\title{
27 Complementing different software environments to mediate the introduction of variable, parameter and unknown
}

\author{
Giuliana Dettori, Simonetta Greco and Enrica Lemut \\ I.M.A., C.N.R., \\ Genova, Italy
}

\begin{abstract}
Understanding the different roles of letters as variables, parameters and unknowns in algebraic expressions and relations is hard for students both when building formulae and when interpreting them; nevertheless it is crucial, since these two activities are the base of algebraic modelling. The parallel use of several software environments and the creation of links between the representations can highlight different aspects of these concepts and lead to both a better understanding and an increased ability to apply them in different situations.
\end{abstract}

Algebra, problem solving, software.

\section{Key words}

\section{Introduction}

Though letters in an algebraic expression always take the place of numbers, the context for the expression determines the level of meaning (Bloedy-Vinner, 1994). Several words are used to indicate the roles of letters-constant, variable, parameter, unknown - each with a precise meaning. Distinguishing these roles is usually hard for students, both when building formulae and when interpreting them, since it requires not only abstraction capabilities, but also different abstractions depending on the role of the letter. These differences are usually not sufficiently emphasised in school in the early use of letters, in textbooks and by teachers, since more attention is usually paid to manipulation skills than to modelling capabilities, and manipulations are independent of the role of the letter. Moreover, unwritten rules are often implicitly applied, such as the use $x, y, z$ and other letters at the end of the alphabet for variables and unknowns, and $a, b, c, k$ and near-by letters for parameters and constants. Such conventions, which are not based on any mathematical reason, can be a source of confusion, since students tend to attribute the same role to objects represented by the group (thus confusing variables and unknowns or constants and parameters), and if a different letter is 
used for the 'unknown' can even fail to recognise an equation they have already solved

On the other hand, a correct use of letters is crucial in the algebraic modelling process. Hence we need to face the issue of how letters should be suitably introduced. In order to prevent students from building misconceptions, we suggest that the use of letters should be associated with their meanings from the beginning, that is, they should be used to nominalise quantities in problem solving, rather than to represent numbers by means of different symbols. Our research, which has involved the analysis of several experiences on the introduction of algebra in schools, has shown that the use of a range of software environments can ease the learning of basic algebraic concepts. In particular, among the software usually available in school computer labs, the spreadsheet has particular potential for clarifying and demonstrating an initial approach to understanding the role of letters in relations, and programming can be useful to for reinforcing this understanding. The use of graphical software can also contribute to 'building up' an understanding of the different meanings. Each of these environments allows a different representation and use of the concepts under consideration. However, unless they are correctly and strongly linked, different representations are not sufficient in themselves for producing a flexible use of learned concepts (see, for example, Dreyfus, 1991). We suggest here that the parallel use of several software environments, in working on the same problem, with attention given to making links between representations and leading students to switch flexibly between them, provides a powerful means for introducing the different meaning of letters. In the following section we present a simple example to illustrate how the parallel use of various, widely available, computer-based representations can be implemented for promoting a 'meanings-based' introduction to the role of letters in algebra.

\section{Parallel use of software environments in algebra - an example}

Let us consider a problem situation concerning the relation between the interest rate $(R)$, the deposit period $(N)$, the capital initially invested $(C)$ and the final capital $(K)$. In this context several problems can be posed, where the entities $K, R$, $N$, and $C$ play different roles. From the wide range of possible problems let us consider the following in which $C$ and $R$ play the role of parameters, $N$ the independent variable and $K$ the dependent variable:

A man deposits 1000 dollars $n$ the bank; he gets an interest of $4 \%$ a year; how much money does he have after any number of years?

Using a spreadsheet, the teacher can guide her students in building a table which contains the number of years in the first column and the corresponding capital's value in the second, writing formulas for their computation (see fig. 1). By observing the table's regularities, the teacher is able to guide her students in:

- Abstracting the concept of variable (Dettori, in press)-though the spreadsheet does not explicitly use algebraic variables but only references to 
cells, two variables ( $K$ and $N$ ), are conceptually present in the two columns and can be deduced by observing the identical structure of formulas for each of the columns.

- Understanding the concept of parameter, which is like a second-order variable, differentiating a problem from the others in a class of problems with the same structure, with its value changing from problem to problem, but behaving as a constant within a given one. A generalisation of the table can be discussed by changing the initial capital or the interest rate. The distinction between absolute and relative references underlines the distinction between parameters and variables.

- Understanding the conceptual notions of independent and dependent variables - the variable corresponding to column $A$ is independent, while that corresponding to column $B$ depends in some way on that in $A$; this dependence can be guessed in the table, though it is not explicit in functional terms. The concept of functional dependence can be deepened by substituting the formulas of column $B$ one into the other, or can be faced again later on by solving a different problem where the dependence is explicit in the formulas used to build the table.

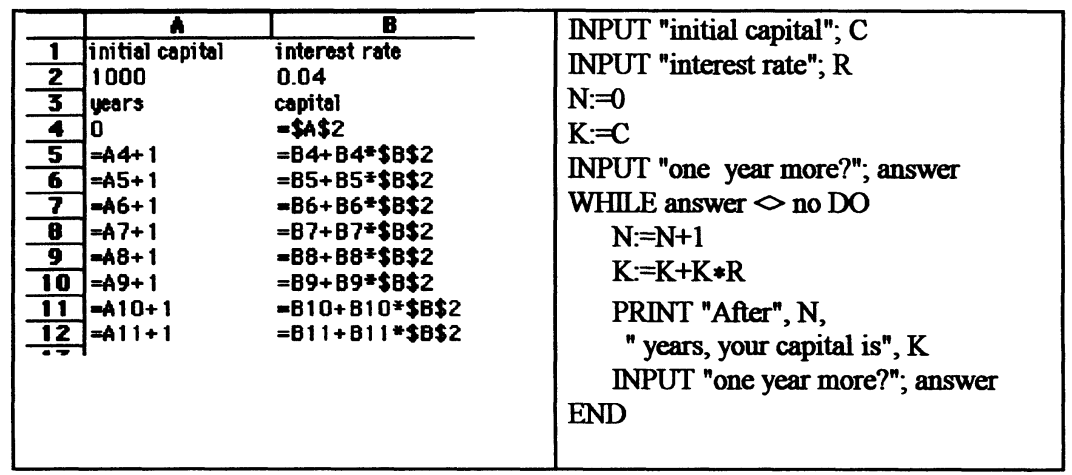

Figure 1

Figure 2

In a programming environment, this problem can be solved in several different ways. Using the experience from constructing and analysing the spreadsheet table, the teacher can now guide her students in developing a program such as that given in figure 2 (in pseudo-Pascal), reinforcing the abstract concepts previously introduced-in particular, as concerns the variables, the instructions to update the number for the year and the amount of capital represent a generalisation of the formulas used in the table. The fact that these expressions summarise the formulas written in the spreadsheet (column by column) highlights the generality of the variables involved; and, at the same time, the fact that each expression actually corresponds to many formulas aids in demonstrating how variables work. In terms of parameters, the use of absolute notation in the spreadsheet 
differentiates them from variables, but can lead to confusion with constants. However, this distinction is instead underlined in the program, since to make the program general, they need to be input by the user.

Through a reformulation of the assignment as 'Compute how many years it takes for the capital to reach a given amount', the same problem situation can now be used to introduce the concept of unknown. The answer can be constructed based on the above table or on the output of the program, simply by looking for a value of $\mathrm{N}$ such that the given condition on $\mathrm{K}$ is satisfied. Here the teacher should point out that now the values in the first column must be regarded as trials made to find the requested solution. This different way of regarding $\mathrm{N}$ highlights the difference between unknown and variable. Since table and program are equivalent under this respect, it is advisable to use only one of them and to complement it with a graphical representation, possibly automatically drawn. The link to stress in considering the table and graph is that of the possibility of reading the graph starting from the $x$ axis $(\mathrm{N}$ as variable) or from the $y$ axis $(\mathrm{N}$ as unknown), which corresponds to reading the table starting from column $A$ or from column B.

\section{Conclusion}

As proposed in the introduction, and illustrated with the example, the parallel use of several software environments and the creation of links between the representations produced their use, can be used to highlight different aspects of abstract concepts. This in turn leading to a better understanding of the concepts and an increased ability to use them in different situations.

\section{References}

Bloedy-Vinner, H. (1994). The Analgebraic Mode of Thinking-The Case of Parameter. In J. da Ponte and J. Matos (eds.) Proceedings of XVIII PME International Conference for the Psychology of Mathematics Education, 2, University of Lisbon, Portugal, 88-95.

Dettori, G., Garuti, R. and Lemut, E. (in press). From Arithmetic to Algebraic Thinking by Using a Spreadsheet. In R. Sutherland (ed.) Algebraic Processes and Structures. Kluwer: Dordrecht, The Netherlands.

Dreyfus, T. (1991). Advanced Mathematical Thinking processes. In D. Tall (ed.) Advanced Mathematical Thinking. Kluwer: Dordrecht, The Netherlands. 